\title{
Heuristic Approach to Establish New Operators via Nano Topology
}

\author{
Lellis Thivagar Maria Antony, Priyalatha Sundharambal Perumal Rajendran \\ School of Mathematics, Madurai Kamaraj University, Madurai (Dt), India \\ Email: mlthivagar@yahoo.co.in, spr.priyalatha@gmail.com
}

How to cite this paper: Antony, L.T.M. and Rajendran, P.S.P. (2017) Heuristic Approach to Establish New Operators via Nano Topology. Applied Mathematics, 8, 1186-1194.

https://doi.org/10.4236/am.2017.88089

Received: June 9, 2017

Accepted: August 27, 2017

Published: August 30, 2017

Copyright $\odot 2017$ by authors and Scientific Research Publishing Inc. This work is licensed under the Creative Commons Attribution International License (CC BY 4.0).

http://creativecommons.org/licenses/by/4.0/

\begin{abstract}
This article presents a new kind of class of all after composed set and fore composed set using the arbitrary binary relation based on nano topological space. We express the notion of nano equality, and nano inclusion and nano power set regarding binary relation based on nano topology. Also, we discuss their properties. Finally, the real life application of network topology is studied.
\end{abstract}

\section{Keywords}

Nano Topology, After Composed Set, Nano Equality, Nano Inclusion, Nano Power Set

\section{Introduction}

Thivagar and Richard [1] introduced a nano topological space with respect to a subset $X$ of an universe, which is defined in terms of lower and upper approximations and boundary region of $X$. This paper introduces and defines a new type of class of all after-composed set and the class of all fore composed set denoted by $\tau_{R}$ and ${ }_{R} \tau$ and used in nano topological space induced by any binary relation. Some of their properties are studied and investigated. We define new operators such as nano equality, nano inclusion and nano power set concerning any binary relation in nano topology. Then, we show the differences and relationships between the notions of ordinary set theory and nano topological space. Finally, we present the application of network topology [2] in nano topology.

\section{Preliminaries}

Definition 2.1. [3] [4] [5] [6] For the pair of approximation space $(\mathcal{U}, R)$ 
where $\mathcal{U}$ is the non-empty finite set of objects called the universe, $R$ be an binary relation on $\mathcal{U}$. Then the set $x R$ is defined as $x R=\{y \in \mathcal{U} \mid x R y\}$ is called as the right neighborhood of an element $x \in \mathcal{U}$.

Definition 2.2. [5] [7] Let $(\mathcal{U}, R)$ be approximation space and $X \subseteq \mathcal{U}$. The subset $X$ is called an after (resp.,fore) composed set if $X$ contains all after (resp.,fore) sets for all elements of its points that is for all $x \in X, x R \subseteq X$ (resp., $R X \subseteq X)$. The class of all after composed sets and fore composed set in $(\mathcal{U}, R)$ is $\tau_{R}=\{X \subseteq \mathcal{U} \mid \forall x \in X ; x R \subseteq X\}$ and ${ }_{R} \tau=\{X \subseteq \mathcal{U} \mid \forall x \in X ; R x \subseteq X\}$.

Definition 2.3. [1] [8] Let $\mathcal{U}$ be a non-empty finite set of objects called the universe $R$ be an equivalence relation on $\mathcal{U}$ named as the indiscerniblity relation. Elements belonging to the same equivalence class are said to be indiscernible with one another. The pair $(\mathcal{U}, R)$ is said to be the approximation space. Let $X \subseteq \mathcal{U}$.

(i) The Lower approximation of $X$ with respect to $R$ is the set of all objects, which can be for certain classified as $X$ with respect to $R$ and it is denoted by $L_{R}(X)$. That is, $L_{R}(X)=\bigcup\{R(x): R(x) \subseteq X\}$, where $R(x)$ denotes the equivalence class determined by $x$.

(ii) The Upper approximation of $X$ with respect to $R$ is the set of all objects, which can be possibly classified as $X$ with respect to $R$ and it is denoted by $U_{R}(X)=\bigcup\{R(x): R(x) \cap X \neq \varnothing\}$.

(iii) The Boundary region of $X$ with respect to $\mathrm{R}$ is the set of all objects which can be classified neither as $X$ nor as not $X$ with respect to $R$ and it is denoted by $B_{R}(X)=U_{R}(X)-L_{R}(X)$.

Definition 2.4. [1] Let $\mathcal{U}$ be the universe, $R$ be an indiscernibility relation on $\mathcal{U}$ and $\tau_{R}(X)=\left\{\mathcal{U}, \varnothing, L_{R}(X), U_{R}(X), B_{R}(X)\right\}$ where $X \subseteq \mathcal{U}$, then $\tau_{R}(X)$ satisfies the following axioms:

(i) $\mathcal{U}$ and $\varnothing \in \tau_{R}(X)$.

(ii) The union of elements of any sub collection of $\tau_{R}(X)$ is in $\tau_{R}(X)$.

(iii) The intersection of the elements of any finite sub collection of $\tau_{R}(X)$ is in $\tau_{R}(X)$.

That is, $\tau_{R}(X)$ forms a topology on $\mathcal{U}$ called as the nano topology on $\mathcal{U}$ with respect to $X$. We call $\left\{\mathcal{U}, \tau_{R}(X)\right\}$ as the nano topological space.

Throughout this paper a binary relation is called as a relation and also after composed set,fore composed set are called as relational topology.

\section{Relational Topology Based on Nano Topological Space}

In this section, we define the after and fore-composed sets based on nano topological space are investigated.

Definition 3.1. Let $\mathcal{U}$ be a non empty finite set of objects called the universe $\mathrm{R}$ be any binary relation on $\mathcal{U}$. The class of all after- (resp., fore-) composed sets in $(\mathcal{U}, R)$ is an ordered pair of approximation space and $\tau_{R}^{*}(X)=\left\{\mathcal{U}, \varnothing, L_{R}(X), U_{R}(X), B_{R}(X)\right\} \quad$ where $X \subseteq \mathcal{U}$ and if lower and upper approximations and boundary region of $X$ is given by 
(i) $L_{R}(X)=\bigcup\left\{G \in \tau_{R}: G \subseteq X\right\}$.

(ii) $U_{R}(X)=\bigcap\left\{H \in{ }_{R} \tau: X \subseteq H\right\}$.

(iii) $B_{R}(X)=U_{R}(X)-L_{R}(X)$.

where $\tau_{R}^{*}(X)$ forms a topology on $\mathcal{U}$ called as the nano topology induced by relational topology on $\mathcal{U}$ with respect to any relation. We call $\left(\mathcal{U}, \tau_{R}^{*}(X)\right)$ as the nano topology induced by relational topology.

Example 3.2. Let $\mathcal{U}=\{a, b, c, d\}$ and $X=\{b\}$ with $R=\{(a, a),(b, b),(c, b),(c, c),(c, d),(d, a)\}$. Then we have $a R=\{a\}, b R=\{b\}$, $c R=\{b, c, d\}, \quad d R=\{a\}$ and $R a=\{a, d\}, R b=\{b, c\}, R c=\{c\}, R d=\{c\}$. Here $\tau_{R}=\{\mathcal{U}, \varnothing,\{a\},\{b\},\{a, b\},\{a, d\},\{b, d\},\{a, b, d\}\}$ and ${ }_{R} \tau=\{\mathcal{U}, \varnothing,\{c\},\{b, c\},\{c, d\},\{b, c, d\},\{a, c, d\}\}$. Then $L_{R}(X)=\{b\}$, $U_{R}(X)=\{b, c\}$ and $B_{R}(X)=\{c\}$. Hence $\tau_{R}^{*}(X)=\{\mathcal{U}, \varnothing,\{b\},\{b, c\},\{c\}\}$.

Proposition 3.3. The class $\tau_{R} /{ }_{R} \tau$ in approximation space $(\mathcal{U}, R)$ in a topology on $\mathcal{U}$.

Proof: We shall prove that $\tau_{R}$ is a topology on $\mathcal{U}$ and similarly for ${ }_{R} \tau$. Clearly $\mathcal{U}$ and $\varnothing$ are after composed sets, then $\mathcal{U}, \varnothing \in \tau_{R}$. Let $A, B \in \tau_{R}$, and let $x \in A \cap B$.Then $x \in A$ and $x \in B$, which implies that $x R \subseteq A$ and $x R \subseteq B$.Thus $x R \subseteq A \cap B$, and then $A \cap B \in \tau_{R}$. Now,let $A_{i} \in \tau_{R} \forall i \in I$.Then $x \in \bigcup A_{i}$ imply that there exist $i_{0} \in I$ such that $x \in A_{i_{0}} \in \bigcup A_{i}$, and hence $x R \subseteq{ }_{i \in I} A_{i_{0}} \subseteq \bigcup_{i \in I} A_{i}$, that is $\bigcup_{i \in I} A_{i} \in \tau_{R}$. Thus $\tau_{R} /{ }_{R} \tau$ is a topology on $\mathcal{U}$.

\section{New Operators in Nano Topology}

In this section we will give the basic deviations for equality $(\simeq, \bar{\sim} \approx)$, inclusion $(\subsetneq, \tilde{\subset}, \tilde{\sim})$ and power set is an ordinary set theoretical operation approach to approximation space in nano topology equipped with relation. The relation represents the basic and necessary concept to define the after and fore composed sets induced by relation over nano topological space.

Definition 4.1. Let $\left(\mathcal{U}, \tau_{R}^{*}(X)\right)$ be nano topological space induced by relational topology. Then the two subsets $X, Y \subseteq \mathcal{U}$ are called as follows:

(i) Nano Lower-equal in $(\mathcal{U}, R)$, written $X \simeq Y$, if $L_{R}(X)=L_{R}(Y)$.

(ii) Nano Upper-equal in $(\mathcal{U}, R)$, written $X \approx Y$, if $U_{R}(X)=U_{R}(Y)$.

(iii) Nano almost equal in $(\mathcal{U}, R)$.written, if $X \approx Y$, if $X \simeq Y$ and $X \approx Y$.

Example 4.2. Let $\mathcal{U}=\{a, b, c, d, e\}$ and $R=\{(a, a),(b, c),(b, d),(c, e),(c, a),(d, d),(d, a),(e, e)\}$, where $a R=\{a\}$, $b R=\{c, d\}, \quad c R=\{e, a\}, d R=\{d, a\}, \quad e R=\{e\}$ and $R a=\{a, c, d\}, R b=\varnothing$, $R c=\{b\}, R d=\{b, d\}, R e=\{c, e\}$. Then $\tau_{R}=\{\mathcal{U}, \varnothing,\{a\},\{e\},\{a, d\},\{a, e\},\{a, c, e\},\{a, d, e\},\{a, c, d, e\}\}$ and ${ }_{R} \tau=\{\mathcal{U}, \varnothing,\{b\},\{b, c\},\{b, c, d\},\{b, c, e\},\{a, b, c, d\},\{b, c, d, e\}\}$. Let $X=\{a, c, d\}$ and $Y=\{a, b, d\}$. Then $L_{R}(X)=\{a, d\}=L_{R}(Y)$ i.e., $X \simeq Y$ and $U_{R}(X)=$ $\{a, b, c, d\}=U_{R}(Y)$ i.e., $X \approx Y$. Thus $X \approx Y$ is equal to $X \simeq Y$ and $X \approx Y$.

Definition 4.3. Let $\left(\mathcal{U}, \tau_{R}^{*}(X)\right)$ be nano topological space induced by relational topology and $X, Y \subseteq \mathcal{U}$. We say that:

(i) $X$ is nano lower-included in $Y$, written $X \subseteq Y$, if $L_{R}(X) \subseteq L_{R}(Y)$. 
(ii) $X$ is nano upper-included in $Y$, written $X \tilde{\subset} Y$, if $U_{R}(X) \subseteq U_{R}(Y)$.

(iii) $X$ is nano included in $Y$, written $X \tilde{\sim} Y$, if $X \tilde{\subset} Y$ and $X \tilde{\subset} Y$.

Example 4.4. Consider $\mathcal{U}=\{a, b, c, d\}$ and $R=\{(a, a),(b, b),(c, b),(c, c),(d, a)\}$, where $a R=\{a\}, \quad b R=\{b\}, c R=\{b, c\}$, $d R=\{a\}$. Then $\tau_{R}=\{\mathcal{U}, \varnothing,\{a\},\{b\},\{a, b\},\{a, d\},\{b, c\},\{a, b, c\},\{a, b, d\}\}$ and $\tau_{R}^{*}=\{\mathcal{U}, \varnothing,\{c\},\{d\},\{a, d\},\{b, c\},\{c, d\},\{a, c, d\},\{b, c, d\}\}$. Let $X=\{b, c\}$ and $Y=\{a, b, d\}$, clearly $X \nsubseteq Y$ and we have $L_{R}(X)=\{b, c\}, L_{R}(Y)=\{a, b, d\}$, $U_{R}(X)=\{b, c\}$ and $U_{R}(Y)=\mathcal{U}$. Then $X \tilde{\subset} Y$ and $X \subset Y$ which implies that $X \underset{\sim}{\sim} Y$.

Definition 4.5. Let $(\mathcal{U}, R)$ be approximation space and $X$ in $\mathcal{U}$. Then the family of all $\tau_{P(R)}(X)=\left\{\mathcal{U}, \varnothing, L_{P(R)}(X), U_{P(R)}(X), B_{P(R)}(X)\right\}$ forms a nano topology on $\mathcal{U}$, which can be defined by

(i) $L_{P(R)}(X)=\{\forall Y \subseteq \mathcal{U}: Y \subset X\}$.

(ii) $U_{P(R)}(X)=\{\forall Y \subseteq \mathcal{U}: Y \subset X\}$.

(iii) $B_{P(R)}(X)=U_{P(R)}(X) \cap L_{P(R)}(X)$.

Then $L_{P(R)}(X), U_{P(R)}(X), B_{P(R)}(X)$ which is known as power set of lower approximation, power set of upper approximation and power set of boundary region of $X$.

Example 4.6. Consider the approximation space $(\mathcal{U}, R)$ in Example 4.4 and let $X=\{a, c\}$ and $P(X)=\{X, \varnothing,\{a\},\{c\}\}$. Then $L_{R}(X)=\{a\}, U_{R}(X)=$ $\{a, c, d\}$ and hence $L_{P(R)}(X)=\{\mathcal{U}, \varnothing,\{a\},\{c\},\{d\},\{c, d\}\}$,

$U_{P(R)}(X)=\{\mathcal{U}, \varnothing,\{a\},\{c\},\{d\},\{a, d\},\{c, d\},\{a, c, d\}\}$ and $B_{P(R)}(X)=\{\mathcal{U}, \varnothing,\{a\},\{c\},\{d\},\{c, d\}\}$.

Clearly, $P(X) \subseteq L_{P(R)}(X), P(X) \subseteq U_{P(R)}(X), P(X) \subseteq B_{P(R)}(X)$.

Proposition 4.7. If $\left(\mathcal{U}, \tau_{R}^{*}(X)\right)$ be nano topological space induced by relational topology and $X, Y, X^{\prime}, Y^{\prime} \subseteq \mathcal{U}$. Then the following conditions are hold:

(i) If $X \simeq Y$, then $(X \cup Y) \simeq X \simeq Y$.

(ii) If $X \approx Y$, then $(X \cup Y) \approx X \approx Y$.

(iii) If $X \simeq X^{\prime}$ and $Y \simeq Y^{\prime}$, then $(X \cup Y) \simeq\left(X^{\prime} \cup Y^{\prime}\right)$.

(iv) If $X \approx X^{\prime}$ and $Y \approx Y^{\prime}$, then $(X \cap Y) \approx X \approx Y^{\prime}$.

Proof:

(i) Let $X \simeq Y$, then $L_{R}(X)=L_{R}(Y)$. But $L_{R}(X \cup Y)=L_{R}(X) \cup L_{R}(Y)$, then $L_{R}(X \cup Y)=L_{R}(X) \cup L_{R}(X)=L_{R}(X) \quad$ and $\quad L_{R}(X \cup Y)=L_{R}(Y) \cup L_{R}(Y)=$ $L_{R}(Y)$. Hence $(X \cup Y) \simeq X \simeq Y$.

(ii) By the proof(ii) is similar way as in proof (i).

(iii) Let $X \simeq X^{\prime}$ and $Y \simeq Y^{\prime}$, then $L_{R}(X)=L_{R}\left(X^{\prime}\right)$ and $U_{R}(Y)=U_{R}\left(Y^{\prime}\right)$. Thus $L_{R}(X) \cup L_{R}(Y)=L_{R}\left(X^{\prime}\right) \cup L_{R}\left(Y^{\prime}\right)$, which implies that $L_{R}(X \cup Y)=$ $L_{R}\left(X^{\prime}\right) \cup L_{R}\left(Y^{\prime}\right)$. Thus $(X \cup Y) \approx\left(X^{\prime} \cup Y^{\prime}\right)$.

iv) By the proof is similar way as in (iii).

Proposition 4.8. Let $(\mathcal{U}, R)$ be approximation space and $\tau_{R}^{*}(X)$ be nano topological space induced by relational topology and $X, Y \subseteq \mathcal{U}$. Then

(i) $X \simeq Y$ if and only if $\left(X^{c}\right) \approx\left(Y^{c}\right)$. 
(ii) If $X \approx \varnothing$ or $Y \approx \varnothing$, then $X \cap Y \approx \varnothing$.

(iii) If $X \simeq \mathcal{U}$ or $Y \simeq \mathcal{U}$, then $(X \cup Y) \simeq \mathcal{U}$.

\section{Proof:}

(i) By the proof is directly from the definition 4.0.

(ii) Let $X \approx \varnothing$ or $Y \approx \varnothing$, then $U_{R}(X)=U_{R}(\varnothing)=\varnothing$ or $U_{R}(Y)=U_{R}(\varnothing)$ $=\varnothing$. Then $U_{R}(X \cap Y)=U_{R}(X) \cap U_{R}(Y)=\varnothing=U_{R}(\varnothing)$, that is $(X \cap Y) \approx \varnothing$.

(iii) By the similar way as in (ii).

Proposition 4.9. Let $\left(\mathcal{U}, \tau_{R}^{*}(X)\right)$ be relational topology based on nano topological space and $X, Y \subseteq \mathcal{U}$. Then

(i) If $X \subseteq Y$, then $X \subseteq Y, X \tilde{\sim} Y$ and $X \underset{\sim}{\sim} Y$.

(ii) $X \subset Y$, and $Y \subset X$ if and only if $X \approx Y$.

(iii) $X \tilde{\subset} Y$ and $Y \tilde{\subset} X$ if and only if $X \simeq Y$.

(iv) $X \tilde{\sim} Y$ and $Y \tilde{\sim} X$ if and only if $X \approx Y$.

\section{Proof:}

(i) The proof is directly from the definition obvious.

(ii) $X \subseteq Y$ and $Y \subset X$ iff $L_{R}(X) \subseteq L_{R}(Y)$ and $L_{R}(Y) \subseteq L_{R}(X)$ iff $L_{R}(Y)=L_{R}(X)$ iff $X \approx Y$.

(iii) and (iv) by similar way as in (ii).

Proposition 4.10. If $(\mathcal{U}, R)$ be approximation space and $\tau_{R}^{*}(X)$ be nano topological space induced by relational topology, and $X, Y, X^{\prime}, Y^{\prime} \subseteq \mathcal{U}$. Then

(i) $X \subset Y$ if and only if $(X \cap Y) \sim X$.

(ii) $X \tilde{\subset} Y$ if and only if $(X \cup Y) \simeq Y$

(iii) $(X \cap Y) \subset X \tilde{\sim}(X \cup Y)$.

(iv) If $X \subseteq Y, X \approx X^{\prime}$ and $Y \approx Y^{\prime}$, then $X^{\prime} \subset Y^{\prime}$.

(v) If $X \subseteq Y, X \simeq X^{\prime}$ and $Y \simeq Y^{\prime}$ and $Y \simeq Y^{\prime}$, then $X^{\prime} \subseteq Y^{\prime}$.

(vi) If $X \subseteq Y, X \approx X^{\prime}$ and $Y \approx Y^{\prime}$, then $X^{\prime} \subseteq Y^{\prime}$.

(vii) If $X^{\prime} \tilde{\subset} X$ and $Y^{\prime} \tilde{\subset} Y$, then $\left(X^{\prime} \cup Y^{\prime}\right) \tilde{\subset}(X \cup Y)$.

(viii) $X^{\prime} \subset X$ and $Y \subset Y$, then $\left(X^{\prime} \cap Y^{\prime}\right) \subset(X \cap Y)$.

\section{Proof:}

(i) Let $X \subseteq Y$ iff $L_{R}(X) \subseteq L_{R}(Y)$ iff $L_{R}(X) \cap L_{R}(Y)$ iff $L_{R}(X \cap Y)=$ $L_{R}(X)$ iff $(X \cap Y) \approx X$.

(ii) By the proof is similar way as in (i).

(iii) Since $L_{R}(X \cap Y)=L_{R}(X) \cap L_{R}(Y)$ and $L_{R}(X \cup Y)=L_{R}(X) \cup L_{R}(Y)$. Then $L_{R}(X \cap Y) \subseteq L_{R}(X)$ and $U_{R}(X) \subseteq(X \cup Y)$ and hence $(X \cap Y) \subseteq$ $X \tilde{\subset}(X \cup Y)$.

(iv) Let $X \subseteq Y, \quad X \approx X^{\prime}$ and $Y \simeq Y^{\prime}$, then $L_{R}(X) \subseteq L_{R}(Y), \quad L_{R}(X)=$ $L_{R}\left(X^{\prime}\right)$ and $L_{R}(Y)=L_{R}\left(Y^{\prime}\right)$. Thus $L_{R}\left(X^{\prime}\right) \subseteq L_{R}\left(Y^{\prime}\right)$ and then $X^{\prime} \subseteq Y^{\prime}$.

(v) and (vi) By the proof is similar way as in(iv).

(vi) Let $X^{\prime} \subseteq X$ and $Y^{\prime} \tilde{\subset} Y$, then $U_{R}\left(X^{\prime}\right) \subseteq U_{R}(X)$ and $U_{R}\left(Y^{\prime}\right) \subseteq$ $U_{R}(Y)$ and hence $U_{R}\left(X^{\prime}\right) \cup U_{R}\left(Y^{\prime}\right) \subseteq U_{R}(X) \cup U_{R}(Y)$ and $U_{R}\left(X^{\prime} \cup Y^{\prime}\right) \subseteq$ $U_{R}(X \cup Y)$. That is $\left(X^{\prime} \cup Y^{\prime}\right) \tilde{\subset}(X \cup Y)$.

(vii) By the proof is similar way as in (vii).

Proposition 4.11. Let $\left(\mathcal{U}, \tau_{R}^{*}(X)\right)$ be nano topological space induced by 
relational topology on $\mathcal{U}$ and $(\mathcal{U}, R)$ be approximation space. Let $X, Y, Z \subseteq \mathcal{U}$. Then

(i) If $X \subseteq Y$ and $X \approx Z$, then $Z \subset Y$.

(ii) If $X \tilde{\subset} Y$ and $X \simeq Z$, then $Z \tilde{\subset} Y$.

(iii) If $X \tilde{\sim} Y$ and $X \approx Z$, then $Z \tilde{\sim} Y$.

Proof: The proof is similar way as in proposition 4.10

Proposition 4.12. Let $\left(\mathcal{U}, \tau_{R}^{*}(X)\right)$ be nano topological space induced by relational topology on $\mathcal{U}$. Then $P(X) \subseteq L_{P(R)}(X), \quad P(X) \subseteq U_{P(R)}(X)$, $P(X) \subseteq B_{P(R)}(X)$.

Proof: Let $Y \in P(X)$, then $Y \subseteq X$ and hence $L_{R}(Y) \subseteq L_{R}(X)$ and $U_{R}(Y) \subseteq U_{R}(X)$. Thus $Y \subset X, Y \tilde{\subset} X$, which implies that $Y \in L_{P(R)}(X)$, $Y \in U_{P(R)}(X)$ and $Y \in B_{P(R)}(X)$. Thus $P(X) \subseteq L_{P(R)}(X)$, $P(X) \subseteq U_{P(R)}(X), B_{P(R)}(X)$.

Proposition 4.13. Let $\left(\mathcal{U}, \tau_{R}^{*}(X)\right)$ be nano topological space induced by relational topology on $\mathcal{U}$ and $(\mathcal{U}, R)$ be approximation space. Let $X, Y \subseteq \mathcal{U}$. Then

(i) If $X \subseteq Y$ then $L_{P(R)}(X) \subseteq L_{P(R)}(Y)$.

(ii) If $X \tilde{\subset} Y$ then $U_{P(R)}(X) \subseteq U_{P(R)}(Y)$.

(iii) If $X \underset{\sim}{\sim} Y$ then $B_{P(R)}(X) \subseteq B_{P(R)}(Y)$.

(iv) If $X \approx Y$ if and only if $L_{P(R)}(X)=L_{P(R)}(Y)$.

(v) If $X \simeq Y$ if and only if $U_{P(R)}(X)=U_{P(R)}(Y)$.

(vi) If $X \approx Y$ if and only if $B_{P(R)}(X)=B_{P(R)}(Y)$.

Proof:

(i) Let $X \subseteq Y$, then $L_{R}(X) \subseteq L_{R}(Y)$. Now let $Z \in L_{P(R)}(X)$, then $Z \subseteq X$, that is $L_{R}(Z) \subseteq L_{R}(X)$. Thus by $L_{R}(Z) \subseteq L_{R}(Y)$ and then $Z \subseteq Y$. Hence $Z \in L_{P(R)}(Y)$ and then $L_{P(R)}(X) \subseteq L_{P(R)}(Y)$.

(ii) and (iii) by the proof is same way as in (i).

(iii) If $X \approx Y$ iff $X \subseteq Y$ and $Y \subset X$ iff $L_{P(R)}(X) \subseteq L_{P(R)}(Y)$ and $L_{P(R)}(Y) \subseteq L_{P(R)}(X)$ iff $L_{P(R)}(X)=L_{P(R)}(Y)$.

(iv) and (vi) by the proof is same way as in (iv).

Proposition 4.14. If $\left(\mathcal{U}, \tau_{R}^{*}(X)\right)$ be relational topology based on nano topological space and $(\mathcal{U}, R)$ be approximation space. Let $X, Y \subseteq \mathcal{U}$. Then

(i) $X \in L_{P(R)}(X), \quad X \in U_{P(R)}(X)$ and $X \in B_{P(R)}(X)$.

(ii) If $X \subseteq Y$, then $L_{P(R)}(X) \subseteq L_{P(R)}(Y), \quad U_{P(R)}(X) \subseteq U_{P(R)}(Y)$ and $B_{P(R)}(X) \subseteq B_{P(R)}(Y)$.

Proof:

(i) Since $\underset{\sim}{\sim} \tilde{\subset}, \tilde{\sim} \sim$ are ordering relations. Then $X \subseteq X, X \tilde{\subset} X$ and $X \tilde{\sim} X$ and hence $X \in L_{P(R)}(X), \quad X \in U_{P(R)}(X)$ and $X \in B_{P(R)}(X)$.

(ii) Let $X \subseteq Y$, then $X \subseteq Y, X \tilde{\sim} Y$ and $X \tilde{\sim} Y$. Hence $L_{P(R)}(X) \subseteq L_{P(R)}(Y)$, $U_{P(R)}(X) \subseteq U_{P(R)}(Y)$ and $B_{P(R)}(X) \subseteq B_{P(R)}(Y)$.

\section{Application}

In this section, we discuss the application of computer networking topology 
refers to the layout or design of the connected devices to applied in nano topological space. It is well known that the computation of lower and upper approximations and boundary region is an approximation space $(\mathcal{U}, R)$. The network topologies [2] can be physical (or) logical. The physical topology of a network refers to the configuration (or) the layout of cables, computers and others.The physical topology means that the physical design of a network including the devices,location and cable installation. Logical topology means that pass the information between the computers. Logical Topology refers to the fact that how data actually transfers in a network as opposed to its design. The main types of physical topologies are bus topology, ring topology, star topology and mesh topology. An applied example of new operators in nano topology in the main types of physical topologies are presented. We will show the above discussion by the following example.

Example 5.1. Let $\mathcal{U}=\left\{x_{1}, x_{2}, x_{3}, x_{4}\right\}$ be a set of four different network topology, where $x_{1}$ is a bus topology, $x_{2}$ is a ring topology, $x_{3}$ is a star topology, $x_{4}$ is a mesh topology and $A=\left\{A_{1}, A_{2}, A_{3}\right\}$ be the attributes of network topology, where $A_{1}=$ The method of transfer data $=\{$ Broadcast, $M u l-$ ticast, Unicast $\}=\left\{a_{1}, b_{1}, c_{1}\right\}, A_{2}=$ Cable Type $=\{$ Twistedpaircable, Thin Coaxialcable, Thick Coaxialcable, Fiber opticcable $\}=\left\{a_{2}, b_{2}, c_{2}, d_{2}\right\}$ and $A_{3}=$ Bandwidth Capacity, $\{10 \mathrm{Mbit} / \mathrm{s}, 10-100 \mathrm{Mbit} / \mathrm{s}, 10 \mathrm{Mbit} / \mathrm{s}-40 \mathrm{Gbit} / \mathrm{s}\}=$ $\left\{a_{3}, b_{3}, c_{3}\right\}$.

Data for Network Topology

\begin{tabular}{cccc}
\hline $\mathcal{U} / A$ & $A_{1}$ & $A_{2}$ & $A_{3}$ \\
\hline$x_{1}$ & $\left\{a_{1}\right\}$ & $\left\{b_{2}, c_{2}\right\}$ & $\left\{a_{3}\right\}$ \\
$x_{2}$ & $\left\{a_{1}\right\}$ & $\left\{c_{2}, d_{2}\right\}$ & $\left\{b_{3}\right\}$ \\
$x_{3}$ & $\left\{a_{1}, b_{1}, c_{1}\right\}$ & $\left\{a_{2}, b_{2}, c_{2}, d_{2}\right\}$ & $\left\{c_{3}\right\}$ \\
$x_{4}$ & $\left\{c_{1}\right\}$ & $\left\{a_{2}, b_{2}, c_{2}, d_{2}\right\}$ & $\left\{c_{3}\right\}$ \\
\hline
\end{tabular}

If

$R=\left\{\left(x_{1}, x_{1}\right),\left(x_{2}, x_{2}\right),\left(x_{3}, x_{3}\right),\left(x_{4}, x_{4}\right),\left(x_{1}, x_{2}\right),\left(x_{1}, x_{3}\right),\left(x_{2}, x_{1}\right),\left(x_{2}, x_{3}\right),\left(x_{4}, x_{3}\right)\right\}$. with $\quad x_{1} R=\left\{x_{1}, x_{2}, x_{3}\right\} ; \quad x_{2} R=\left\{x_{1}, x_{2}, x_{3}\right\} ; \quad x_{3} R=\left\{x_{3}\right\} ; \quad x_{4} R=\left\{x_{3}, x_{4}\right\} \quad$ and $R x_{1}=\left\{x_{1}, x_{2}\right\} ; R x_{2}=\left\{x_{1}, x_{2}\right\} ; R x_{3}=\mathcal{U} ; R x_{4}=\left\{x_{4}\right\} \quad$ and $\quad$ we get $\tau_{R}=\left\{\mathcal{U}, \varnothing,\left\{x_{3}\right\},\left\{x_{1}, x_{2}, x_{3}\right\}\right\},{ }_{R} \tau=\left\{\mathcal{U}, \varnothing,\left\{x_{4}\right\},\left\{x_{1}, x_{2}\right\},\left\{x_{1}, x_{2}, x_{4}\right\}\right\}$. Now we have to calculate the nano approximate equal, nano inclusion, nano power set as the following example: Let $X=\left\{x_{1}, x_{2}, x_{3}\right\}$ and $Y=\mathcal{U}$. Then $L_{R}(X)=$ $\left\{x_{1}, x_{2}, x_{3}\right\} \quad$ and $L_{R}(Y)=\left\{x_{1}, x_{2}, x_{3}\right\} \quad$ and $U_{R}(X)=\mathcal{U}=U_{R}(Y)$. Hence $L_{R}(X)=L_{R}(Y)$ that is $X \approx Y$ and $U_{R}(X)=U_{R}(Y)$ that is $X \simeq Y$. Thus $X \approx Y$ although $X \neq Y$.

Next, we show that the nano inclusion. Let $X=\left\{x_{3}, x_{4}\right\}$ and $Y=\left\{x_{1}, x_{2}, x_{3}\right\}$ $\subseteq \mathcal{U}$. Clearly, $X \nsubseteq Y$ we have $L_{R}(X)=\left\{x_{3}\right\}$ and $L_{R}(Y)=\left\{x_{1}, x_{2}, x_{3}\right\}$ and $U_{R}(X)=\mathcal{U}=U_{R}(Y)$. Hence $L_{R}(X) \subseteq L_{R}(Y)$ that is $X \subseteq Y$ and 
$U_{R}(X) \subseteq U_{R}(Y)$ that is $X \tilde{\subset} Y$.Thus $X \underset{\sim}{\sim} Y$ although $X \nsubseteq Y Y$. Then we have to prove that nano power set.Let $X=\left\{x_{1}, x_{3}\right\}$ Then $L_{R}(X)=\left\{x_{3}\right\}$ and $U_{R}(X)=\mathcal{U}$ and $P(X)=\left\{X, \varnothing,\left\{x_{1}\right\},\left\{x_{3}\right\}\right\}$. We obtain that

$$
\begin{aligned}
L_{P(R)}(X)= & \left\{\mathcal{U}, \varnothing,\left\{x_{1}\right\},\left\{x_{2}\right\},\left\{x_{3}\right\},\left\{x_{4}\right\},\left\{x_{1}, x_{2}\right\},\left\{x_{1}, x_{3}\right\},\left\{x_{1}, x_{4}\right\},\right. \\
& \left.\left\{x_{2}, x_{3}\right\},\left\{x_{2}, x_{4}\right\},\left\{x_{1}, x_{2}, x_{4}\right\},\left\{x_{2}, x_{3}, x_{4}\right\},\left\{x_{3}, x_{4}\right\}\right\} \\
U_{P(R)}(X)= & \left\{\mathcal{U}, \varnothing,\left\{x_{1}\right\},\left\{x_{2}\right\},\left\{x_{3}\right\},\left\{x_{4}\right\},\left\{x_{1}, x_{2}\right\},\left\{x_{1}, x_{3}\right\},\left\{x_{1}, x_{4}\right\},\left\{x_{2}, x_{3}\right\}, \quad\right. \text { and } \\
& \left.\left\{x_{2}, x_{4}\right\},\left\{x_{3}, x_{4}\right\},\left\{x_{1}, x_{2}, x_{3}\right\},\left\{x_{1}, x_{2}, x_{4}\right\},\left\{x_{2}, x_{3}, x_{4}\right\},\left\{x_{1}, x_{3}, x_{4}\right\}\right\}, \\
B_{P(R)}(X)= & \left\{\mathcal{U}, \varnothing,\left\{x_{1}\right\},\left\{x_{2}\right\},\left\{x_{3}\right\},\left\{x_{4}\right\},\left\{x_{1}, x_{2}\right\},\left\{x_{1}, x_{3}\right\},\left\{x_{1}, x_{4}\right\},\right. \\
& \left.\left\{x_{2}, x_{3}\right\},\left\{x_{2}, x_{4}\right\},\left\{x_{1}, x_{2}, x_{4}\right\},\left\{x_{2}, x_{3}, x_{4}\right\},\left\{x_{3}, x_{4}\right\}\right\}
\end{aligned}
$$

Then the observations are follows:

(i) If two different set $X$ and $Y$ which are not equal that is $X \neq Y$ in ordinary set theory can be approximate equal in nano topological space that is $L_{R}(X)=L_{R}(Y)$ i.e., $X \simeq Y, U_{R}(X)=U_{R}(Y)$ i.e., $X \approx Y$ thus $X \approx Y$.

(ii) If the nano inclusion of sets does not imply the inclusion of sets.

(iii) If the concept of power set $\mathrm{P}(\mathrm{X})$ in ordinary set theory differs from the concept of nano power set in nano topological space and it is clear that $L_{P(R)}(X) \notin P(X), \quad U_{P(R)}(X) \notin P(X), \quad B_{P(R)}(X) \notin P(X)$.

\section{Conclusion}

This paper systematically studied that any kind of binary relation can be used to relational topology induced by nano topological space. The main aspect of this paper is to introduce nano topology by using the class of all after-composed set and the class of all fore-composed set. Now, we define the new operators in nano equality, nano inclusion and nano power set are now clear with respect to any relation in nano topology. We have proved that there exist similar properties. We will investigate the application of network topology.

\section{Acknowledgements}

The authors are grateful to the anonymous referee for a careful checking of the details and for helpful comments that improved this paper and funding for this work was supported by University Grants Commission, New Delhi.

\section{References}

[1] Thivagar, M.L. and Richard, C. (2013) On Nano Forms of Weakly Open Sets. International journal of Mathematics and statistics Invention, 1, 31-37.

[2] Martin, M.J. (2000) Understanding the Network a Practical Guide to Internet Working. New Riders Publishing, 11, 341-356.

[3] Abo-Tabl, E.A. (2011) A Comparison of Two Kinds Definitions of Rough Approximation Based on Similarity Relation. Information Sciences, 181, 2587-2596. https://doi.org/10.1016/j.ins.2011.01.007

[4] Abo-Tabl, E.A. (2013) Rough Sets and Topological Spaces Based on Similarity. International Journal of Machine Learning and Cybernetics, 4, 451-458. https://doi.org/10.1007/s13042-012-0107-7 
[5] Dubois, D. and Prade, H. (1990) Rough Fuzzy Sets and Fuzzy Rough Sets. International Journal of General Systems, 17, 191-208. https://doi.org/10.1080/03081079008935107

[6] Zhu, W. (2009) Relationship between Generalized Rough Sets Based on Binary Relation and Covering. Journal of Information Science, 2, 210-225. https://doi.org/10.1016/j.ins.2008.09.015

[7] Lin, T.Y. (1998) Granular Computing on Binary Relation, I: Data Mining and Neighborhood Systems. Rough Sets in Knowledge Discovery Physica-Verlag, 2, 107-121.

[8] Pawlak, Z. (1982) Rough Sets. International journal of computer and Information Sciences, 2, 341-356.

Submit or recommend next manuscript to SCIRP and we will provide best service for you:

Accepting pre-submission inquiries through Email, Facebook, LinkedIn, Twitter, etc. A wide selection of journals (inclusive of 9 subjects, more than 200 journals)

Providing 24-hour high-quality service

User-friendly online submission system

Fair and swift peer-review system

Efficient typesetting and proofreading procedure

Display of the result of downloads and visits, as well as the number of cited articles Maximum dissemination of your research work

Submit your manuscript at: http://papersubmission.scirp.org/

Or contact am@scirp.org 